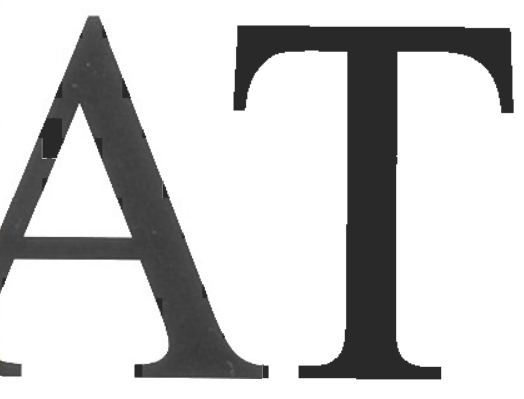

Conferencias, seminarios y trabajos de Matemática

IS SN: $1515-4904$

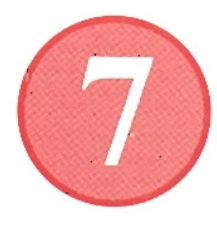

Primeras Jornadas

sobre Ecuaciones

Diferenciales,

Optimización y

Análisis Numérico

Primera Parte 
MAT

\section{SERIE A: CONFERENCIAS, SEMINARIOS Y TRABAJOS DE MATEMÁTICA}

No. 7

\section{PRIMERAS JORNADAS SOBRE ECUACIONES DIFERENCIALES, OPTIMIZACIÓN Y ANÁLISIS NUMÉRICO}

Primera Parte

Domingo A. Tarzia (Ed.)

\section{INDICE}

Adriana B. Verdiell - María C. Maciel - Susana L. Orofino - Tatiana I. Gibelli, "A survey of the spectral gradient method”, 1-14.

María F. Natale - Domingo A. Tarzia, "An integral equation in order to solve a one-phase Stefan problem with nonlinear thermal conductivity”, 15-24.

María C. Sanziel - Domingo A. Tarzia, "Optimization on the heat flux in a mixed elliptic problem with temperature constraints”, 25-30.

Claudia M. Gariboldi - Domingo A. Tarzia, "A new proof of the convergence of distributed optimal controls on the internal energy in mixed elliptic problems”, 3142. 


\title{
OPTIMIZATION OF THE HEAT FLUX IN A MIXED ELLIPTIC PROBLEM WITH TEMPERATURE CONSTRAINTS
}

\author{
María Cristina Sanziel ${ }^{(1)}$ - Domingo A. Tarzia ${ }^{(2)}$ \\ (1) Consejo de Investigaciones de la Univ. Nacional de Rosario \\ Inst.de Matemática B. Levi - Fac.de Cs. Exactas, Ingeniería y Agrimensura, U.N.R. \\ Av. Pellegrini 250, (2000) Rosario, Argentina. \\ E-mail: sanziel@fceia.unr.edu.ar \\ (2) Dpto.de Matemática - CONICET, Fac.de Cs. Empresariales, Univ.Austral \\ Paraguay 1950, (S2000FZF) Rosario, Argentina \\ E-mail: Domingo.Tarzia@fce.austral.edu.ar
}

\begin{abstract}
For a steady-state heat conduction problem in a poligonal domain $\Omega \subset \mathbb{R}^{\mathrm{n}}$, with heat flux condition in a portion of the boundary, $\Gamma_{2}$, and a Fourier type condition in the rest of the boundary, $\Gamma_{1}$, we obtain the minimum total heat flux on $\Gamma_{2}$, so that the whole material is in the solid phase. For this purpose we use the finite element method in order to convert the optimization problem into a linear programming problem.
\end{abstract}

Key Words: Mixed Elliptic Problem, Steady-State Stefan Problem, Finite Element Method, Linear Programming Problem.

AMS Subject Classification: 65K10, 49K20, 35J85, 65N30

\section{Introduction}

We consider a steady-state heat conduction problem in a material $\Omega$ which occupies a poligonal bounded domain in $\mathbb{R}^{\mathrm{n}}$, with boundary $\Gamma=\Gamma_{1} \cup \Gamma_{2}$ (with meas $\left(\Gamma_{1}\right)>0$ and meas $\left.\left(\Gamma_{2}\right)>0\right)$. We impose a Newton law with a transfer coefficient $\alpha>0$ and an exterior temperature $\mathrm{b}>0$ on $\Gamma_{1}$, and an outcoming heat flux $\mathrm{q}>0$ on $\Gamma_{2}$. We assume, without loss of generality, that the phase-change temperature of the material is $0^{\circ} \mathrm{C}$.

This problem was studied in [TaTa] and it was stablished that if the heat flux q is between a minimum flux $\mathrm{q}_{m}$ and a maximum flux $\mathrm{q}_{\mathrm{M}}$, which are functions of the coefficient $\alpha$ and the temperature b, then there is a steady-state two phase Stefan Problem, that is the temperature is of non-constant sign in $\Omega$.

In [GoTa1] a thermic flux optimization problem was solved: the maximization of the output heat flux on a portion of the boundary domain, $\Gamma_{2}$, while on the other portion, $\Gamma_{1}$, the 
distribution of the temperature was fixed. The maximization was carried out under the condition that there is no phase change.

In [GoTa2] the maximum heat total flux on $\Gamma_{2}$ was found, such that the temperature is positive in the whole domain $\Omega$ considering a boundary Fourier type condition on $\Gamma_{1}$.

Following the ideas of these papers, the goal of the present work is to minimize the total heat flux on the boundary $\Gamma_{2}$ so that all the material is in the solid phase. In order to solve this minimization problem we will use the finite element method and we will obtain a linear programming problem.

We remark that all the results of this work are still valid if we consider that the boundary $\Gamma$ of the domain $\Omega$ is the union of three portions, $\Gamma_{1}, \Gamma_{2}$ and $\Gamma_{3}$, such that on $\Gamma_{1}$ and $\Gamma_{2}$ there exist the same conditions stated above, and $\Gamma_{3}$ is a wall impermeable to heat.

In Section II we present the mathematical model of the minimization problem and in Section III we discretize it and a linear programming problem must be solved in order to obtain the solution.

\section{Mathematical Model of the Problem}

If $\theta$ represents the temperature in $\Omega$ and we define the function $\mathrm{u}=\mathrm{k}_{l} \theta^{+}-\mathrm{k}_{s} \theta^{-}$, where $\mathrm{k}_{i}$ is the conductivity of the phase $i$ ( $i=l$ for the liquid and $i=\mathrm{s}$ for the solid), then the following equations represent the mathematical model of the corresponding steady-state heat conduction problem [Du, Ta]:

$$
\begin{aligned}
& \Delta \mathbf{u}=0 \\
& -\left.\frac{\partial \mathbf{u}}{\partial \mathrm{n}}\right|_{\Gamma_{1}}=\alpha(\mathbf{u}-B), \quad-\left.\frac{\partial \mathbf{u}}{\partial \mathrm{n}}\right|_{\Gamma_{2}}=q
\end{aligned}
$$

where $\mathrm{B}=\mathrm{k}_{l} \mathrm{~b}>0$ and $\mathrm{b}$ is the exterior temperature.

We want to minimize the total heat flux on $\Gamma_{2}$ with the constraint that the whole material is in the solid phase. In other words, the problem is

$$
\text { Find } \mathbf{q}^{*} \text { such that } \mathbf{J}\left(\mathbf{q}^{*}\right) \underset{\mathrm{u}}{=} \operatorname{Inf}_{\substack{\text { in } \Omega}} \mathbf{J}(\mathbf{q})
$$

where

$$
\mathbf{J}(\mathbf{q})=\int_{\Gamma_{2}} \mathrm{q} d \gamma, \mathrm{q} \in \mathrm{L}^{2}\left(\Gamma_{2}\right)
$$

The variational formulation of the problem (1) is given by 


$$
\mathrm{a}_{\alpha}(\mathrm{u}, \mathrm{v})=\mathrm{L}_{\alpha}(\mathrm{v}), \quad \forall \mathrm{v} \in \mathrm{V}, \mathrm{u} \in \mathrm{V}
$$

where

$$
\begin{aligned}
& \mathrm{a}_{\alpha}(\mathrm{u}, \mathrm{v})=\mathrm{a}(\mathrm{u}, \mathrm{v})+\underset{\Gamma_{1}}{\alpha \int_{1} \mathrm{uv} \mathrm{d} \gamma}, \quad \mathrm{L}_{\alpha}(\mathrm{v})=\mathrm{L}(\mathrm{v})+\underset{\Gamma_{1}}{\alpha \mathrm{B} \int \mathrm{v} d \gamma} \\
& \mathrm{a}(\mathrm{u}, \mathrm{v})=\int_{\Omega} \nabla \mathrm{u} \nabla \mathrm{vdx}, \quad \mathrm{L}(\mathrm{v})=-\int_{\Gamma_{2}} \mathrm{qv} \mathrm{d} \gamma, \mathrm{V}=\mathrm{H}^{1}(\Omega) .
\end{aligned}
$$

\section{The Discrete Problem and the Linear Programming Problem}

We construct a regular triangulation $\tau_{\mathrm{h}}$, of the poligonal domain $\Omega$ with Lagrange triangles of type 1 , with afin equivalent finit elements of class $C^{\circ}$, and we approach the space $\mathrm{V}$ by [Ci1]:

$$
\mathrm{V}_{\mathrm{h}}=\left\{\mathrm{v}_{\mathrm{h}} \in \mathrm{C}^{0}(\bar{\Omega}) /\left.\mathrm{v}_{\mathrm{h}}\right|_{\mathrm{T}} \in \mathrm{P}_{1}(\mathrm{~T}), \forall \mathrm{T} \in \tau_{\mathrm{h}}\right\}
$$

where $\mathrm{P}_{1}$ is the set of the polynomials of degree $\leq 1$.

The approximate variational problem consists in finding $\mathrm{u}_{\mathrm{h} \alpha} \in \mathrm{V}_{\mathrm{h}}$ so that

$$
\mathrm{a}_{\alpha}\left(\mathrm{u}_{\mathrm{h} \alpha}, \mathrm{v}_{\mathrm{h}}\right)=\mathrm{L}_{\alpha}\left(\mathrm{v}_{\mathrm{h}}\right), \forall \mathrm{v}_{\mathrm{h}} \in \mathrm{V}_{\mathrm{h}} .
$$

We call $\boldsymbol{N}$ the total number of nodes of the triangulation, $\mathrm{r}$ is the number of nodes on the portion of the boundary $\Gamma_{1}$ and $\mathrm{p}$ is the number of nodes on $\Gamma_{2}$.

Let $\left\{\omega_{i}\right\}_{i=1}^{N}$ a basis of the space $\mathrm{V}_{\mathrm{h}}$. We can think the basis as

$$
\left\{\omega_{i}\right\}_{i=1}^{N}=\left\{\omega_{i}^{1}\right\}_{i=1}^{r} \cup\left\{\omega_{i}^{\Omega}\right\}_{i=r+1}^{N-p} \cup\left\{\omega_{i}^{2}\right\}_{i=\mathrm{N}-p+1}^{N}
$$

where we denote $\omega_{i}^{\mathrm{j}}$ the function whose value is 1 on the node $\mathrm{N}_{i}$, of the boundary $\Gamma_{1}$ if $\mathrm{j}=1$, of the boundary $\Gamma_{2}$ if $\mathrm{j}=2$ or of the interior of the domain $\Omega$ if $\mathrm{j}=\Omega$, and whose values are zero in the other nodes. Then we have

$$
\mathrm{u}_{\mathrm{h} \alpha}=\sum_{\mathrm{i}=1}^{r} \mathrm{u}_{\mathrm{i}}^{1} \omega_{i}^{1}+\sum_{\mathrm{i}=r+1}^{N-\mathrm{p}} \mathrm{u}_{\mathrm{i}}^{\Omega} \omega_{i}^{\Omega}+\sum_{\mathrm{i}=N-\mathrm{p}+1}^{N} \mathrm{u}_{\mathrm{i}}^{2} \omega_{i}^{2}
$$

where $\mathrm{u}_{\mathrm{i}}^{1}(\mathrm{i}=1, \ldots, \mathrm{r}), \mathrm{u}_{\mathrm{i}}^{\Omega}(\mathrm{i}=\mathrm{r}+1, \ldots, N-\mathrm{p})$ and $\mathrm{u}_{\mathrm{i}}^{2}(\mathrm{i}=N-\mathrm{p}+1, \ldots, N)$ are the real unknown values at the corresponding nodes.

With the expresion (5) and considering $\mathrm{v}_{\mathrm{h}}=\omega_{i}^{\mathrm{j}}$ in (4), we get the following system of linear equations 


$$
\begin{aligned}
& \boldsymbol{A}_{1} \mathbf{u}^{1}+\boldsymbol{A}_{2} \mathbf{u}^{\Omega}+\boldsymbol{A}_{3} \mathbf{u}^{2}=\mathbf{b}^{\boldsymbol{\alpha}} \\
& \boldsymbol{A}_{4} \mathbf{u}^{1}+\boldsymbol{A}_{5} \mathbf{u}^{\Omega}+\boldsymbol{A}_{6} \mathbf{u}^{2}=\mathbf{0} \\
& \boldsymbol{A}_{7} \mathbf{u}^{1}+\boldsymbol{A}_{8} \mathbf{u}^{\Omega}+\boldsymbol{A}_{9} \mathbf{u}^{2}=\mathbf{b}(\boldsymbol{q})
\end{aligned}
$$

where the matrices $\boldsymbol{A}_{1}, \ldots, \boldsymbol{A}_{9}$ are given by:

$$
\begin{aligned}
& \boldsymbol{A}_{1}=\left(a_{i j}^{1}\right) \in \boldsymbol{R}^{\boldsymbol{r} \boldsymbol{x} \boldsymbol{r}}, \quad a_{i j}^{1}=\mathrm{a}_{\alpha}\left(\omega_{j}^{1}, \omega_{i}^{1}\right), \quad \boldsymbol{A}_{2}=\left(a_{i j}^{2}\right) \in \boldsymbol{R}^{\boldsymbol{r} \boldsymbol{x} \boldsymbol{N}-(\boldsymbol{p}+\boldsymbol{r})}, a_{i j}^{2}=\mathrm{a}\left(\omega_{j}^{\Omega}, \omega_{i}^{1}\right), \\
& \boldsymbol{A}_{3}=\left(a_{i j}^{3}\right) \in \boldsymbol{R}^{\boldsymbol{r} \boldsymbol{x} \boldsymbol{p}}, \quad a_{i j}^{3}=\mathrm{a}\left(\omega_{j}^{2}, \omega_{i}^{1}\right), \quad \boldsymbol{A}_{4}=\left(a_{i j}^{4}\right) \in \boldsymbol{R}^{N-(\boldsymbol{p}+\boldsymbol{r}) \boldsymbol{x} \boldsymbol{r}}, a_{i j}^{4}=\mathrm{a}\left(\omega_{j}^{1}, \omega_{i}^{\Omega}\right), \\
& \boldsymbol{A}_{5}=\left(a_{i j}^{5}\right) \in \boldsymbol{R}^{N-(p+r) \times N-(p+r)}, \quad a_{i j}^{5}=\mathrm{a}\left(\omega_{j}^{\Omega}, \omega_{i}^{\Omega}\right), \\
& \boldsymbol{A}_{6}=\left(a_{i j}^{6}\right) \in \boldsymbol{R}^{N-(\boldsymbol{p}+\boldsymbol{r}) \times \boldsymbol{p}}, a_{i j}^{6}=\mathrm{a}\left(\omega_{j}^{2}, \omega_{i}^{\Omega}\right), \quad \boldsymbol{A}_{7}=\left(a_{i j}^{7}\right) \in \boldsymbol{R}^{\boldsymbol{p} \times \boldsymbol{r}}, a_{i j}^{7}=\mathrm{a}\left(\omega_{j}^{1}, \omega_{i}^{2}\right), \\
& \boldsymbol{A}_{8}=\left(a_{i j}^{8}\right) \in \boldsymbol{R}^{\boldsymbol{p} \times \boldsymbol{r}}, a_{i j}^{8}=\mathrm{a}\left(\omega_{j}^{\Omega}, \omega_{i}^{2}\right), \boldsymbol{A}_{9}=\left(a_{i j}^{9}\right) \in \boldsymbol{R}^{\boldsymbol{p} \times \boldsymbol{p}}, a_{i j}^{9}=\mathrm{a}\left(\omega_{j}^{2}, \omega_{i}^{2}\right), \\
& \mathbf{u}^{1} \in \boldsymbol{R}^{r}, \quad \mathbf{u}^{2} \in \boldsymbol{R}^{\boldsymbol{p}}, \quad \mathbf{u}^{\Omega} \in \boldsymbol{R}^{N-(p+r)}
\end{aligned}
$$

and

$$
\mathbf{b}^{\alpha}=\left(\mathrm{b}_{i}^{\alpha}\right)_{i=1}^{r} \in \boldsymbol{R}^{r}, \quad \mathbf{b}(q)=\left(\mathrm{b}_{i}(q)\right)_{i=N-\mathrm{p}+1}^{N} \in \boldsymbol{R}^{p},
$$

with

$$
\mathrm{b}_{i}^{\alpha}=\alpha B \int_{\Gamma_{1}} \omega_{i}^{1} \mathrm{~d} \gamma, \quad \mathrm{b}_{i}(\boldsymbol{q})=-\int_{\Gamma_{2}} \mathrm{q} \omega_{i}^{2} \mathrm{~d} \gamma
$$

The system (6) can be expressed as follows

$$
A \mathbf{u}=\boldsymbol{b},
$$

where $\boldsymbol{A} \in \boldsymbol{R}^{\boldsymbol{N} \boldsymbol{N}}$ is the finite element matrix , $\boldsymbol{A}=\left(\begin{array}{lll}\boldsymbol{A}_{1} & \boldsymbol{A}_{2} & \boldsymbol{A}_{3} \\ \boldsymbol{A}_{4} & \boldsymbol{A}_{5} & \boldsymbol{A}_{6} \\ \boldsymbol{A}_{7} & \boldsymbol{A}_{8} & \boldsymbol{A}_{9}\end{array}\right)$

and

$$
\mathbf{u}=\left(\mathbf{u}^{1}, \mathbf{u}^{\Omega}, \mathbf{u}^{2}\right) \in \boldsymbol{R}^{N}, \quad \boldsymbol{b}=\left(\mathbf{b}^{\alpha}, \mathbf{0}, \mathbf{b}(q)\right) \in \boldsymbol{R}^{N}
$$

We obtain the elements $\mathrm{b}_{i}^{\alpha}$ and $\mathrm{b}_{i}(\boldsymbol{q})$ through a numerical computation of the corresponding integrals, for example by using the trapezoidal rule. For this purpose we consider that the curve $\Gamma_{i}(i=1,2)$ can be decomposed by 


$$
\Gamma_{i}=\stackrel{\mathrm{S}}{j=1} \Gamma_{i}^{j}, \quad \text { with } s=\mathrm{p}-1 \text { if } \Gamma_{i} \text { is open or } \quad s=\mathrm{p} \text { if } \Gamma_{i} \text { is closed }
$$

and

$$
\int_{\Gamma_{i}} f \mathrm{~d} \gamma=\sum_{j=1}^{s} \int_{\Gamma_{i}^{j}} f \mathrm{~d} \gamma \approx \sum_{j=1}^{s} \frac{1}{2}\left|\Gamma_{i}^{j}\right|\left(f\left(\mathcal{N}_{j}\right)+f\left(\mathcal{N}_{j+1}\right)\right),
$$

where we have denoted with $\Gamma_{i}^{j}$ the portion of the curve $\Gamma_{i}$ whose limit points are the nodes $\mathcal{N}_{j}$ and $\mathcal{N}_{j+1}$ and we have denoted with $\left|\Gamma_{i}^{j}\right|$ the measure of that portion of the curve.

In this way the linear system (7) becomes in

$$
A \mathbf{u}=M \widetilde{q}+\widetilde{b}
$$

where :

- $\widetilde{\boldsymbol{q}} \in \boldsymbol{R}^{\boldsymbol{p}}$ and $\widetilde{\boldsymbol{q}}_{\boldsymbol{i}}$ is the value of the flux $\boldsymbol{q}$ in the node $\mathcal{N}_{N-\mathrm{p}+\mathrm{i}}$,

- $\widetilde{\boldsymbol{b}} \in \boldsymbol{R}^{N}, \widetilde{\boldsymbol{b}_{i}}$ approaches the value of $\mathrm{b}_{i}^{\alpha}$ for $\mathrm{i}=1 \ldots \mathrm{r}$ and $\widetilde{\boldsymbol{b}_{i}}=0$ for $\mathrm{i}=\mathrm{r}+1, \ldots, \mathrm{N}$,

- $M=\left(\begin{array}{c}M^{1} \\ M^{2}\end{array}\right) \in R^{N \times p}, \quad M^{1} \in R^{(N-p) \times p}$ is a zero matrix,

$\boldsymbol{M}^{\mathbf{2}}=\left(m_{i i}\right)_{i=N-p+1}^{N} \in \boldsymbol{R}^{\boldsymbol{p} \times \boldsymbol{p}}$ is a diagonal matrix , with

$$
\begin{array}{ll}
\mathrm{m}_{i i}= & \text { if } i=N-p+1 \text { or } i=N, \\
-\frac{1}{2}\left(\left|\Gamma_{2}^{i-1}\right|+\left|\Gamma_{2}^{i}\right|\right) & \text { if } i=N-p+2, \ldots, N-1,
\end{array}
$$

if $\Gamma_{2}$ is an open curve, or

$$
\mathrm{m}_{i i}=-\frac{1}{2}\left(\left|\Gamma_{2}^{i-1}\right|+\left|\Gamma_{2}^{i}\right|\right) \quad \text { if } i=N-p+1, \ldots, N
$$

if $\Gamma_{2}$ is a closed curve, (here we have considered $\Gamma_{2}^{N-p}=\Gamma_{2}^{N}$ ), and the i-th component of the vector $\boldsymbol{M} \widetilde{\boldsymbol{q}}$ approaches the value of $\mathrm{b}_{i}(\boldsymbol{q})$ i.e. $(\boldsymbol{M} \widetilde{\boldsymbol{q}})_{i} \approx \mathrm{b}_{i}(\boldsymbol{q})$.

After all these considerations, the optimization problem (2) is transformed into the following linear programming problem :

$$
\underset{\mathrm{u} \in \boldsymbol{U}}{\operatorname{Minimize}} \hat{F}(\widetilde{\boldsymbol{q}})=<\boldsymbol{T}_{\Gamma_{2}, \widetilde{\boldsymbol{q}}}>_{\boldsymbol{R}^{p}}
$$

where

$$
\frac{1}{2}\left(\left|\Gamma_{2}^{1}\right|,\left(\left|\Gamma_{2}^{1}\right|+\left|\Gamma_{2}^{2}\right|\right), \ldots,\left(\left|\Gamma_{2}^{\mathrm{p}-2}\right|+\left|\Gamma_{2}^{\mathrm{p}-1}\right|\right),\left|\Gamma_{2}^{\mathrm{p}-1}\right|\right) \quad \text { if } \Gamma_{2} \text { is open, }
$$

$T_{\Gamma_{2}}=$

$$
\frac{1}{2}\left(\left(\left|\Gamma_{2}^{\mathrm{p}}\right|+\left|\Gamma_{2}^{1}\right|\right),\left(\left|\Gamma_{2}^{1}\right|+\left|\Gamma_{2}^{2}\right|\right), \ldots,\left(\left|\Gamma_{2}^{\mathrm{p}-1}\right|+\left|\Gamma_{2}^{\mathrm{p}}\right|\right)\right) \quad \text { if } \Gamma_{2} \text { is closed, }
$$


$<., .>{ }_{\boldsymbol{R}^{p}}$ is the usual inner product in $\boldsymbol{R}^{\boldsymbol{p}}$ and the set $\boldsymbol{U}$ is defined by

$$
U=\left\{\widetilde{q} \in R^{p}: C \widetilde{q} \leq d, C \in R^{N x p}, d \in R^{N}\right\}
$$

with $\boldsymbol{C}=\boldsymbol{A}^{-1} \boldsymbol{M}$ and $\quad \boldsymbol{d}=-\boldsymbol{A}^{-1} \widetilde{\boldsymbol{b}}$.

Taking into account that $\hat{F}(\widetilde{q}) \geq 0, \forall \widetilde{\boldsymbol{q}} \geq 0$ it results that the linear programming problem (8) admits at least one solution [Ci2].

We construct a programm in MATLAB and we get the solution of the problem (8) for some different domains. From these numerical results we can guess that the minimum optimum flux is given by $q^{*}=-\left.\frac{\partial \mathbf{u}^{*}}{\partial \mathrm{n}}\right|_{\Gamma_{2}}$ where $\mathbf{u}^{*}$ is the solution of the following elliptic problem

$$
\begin{array}{ll}
\Delta \mathbf{u}^{*}=0 & \text { in } \Omega \\
\left.\mathbf{u}^{*}\right|_{\Gamma_{1}}=0, & \left.\frac{\partial \mathbf{u}^{*}}{\partial \mathrm{n}}\right|_{\Gamma_{1}}=\alpha B .
\end{array}
$$

\section{References}

[Ci1] P.G. CIARLET, "The finite element method for elliptic problems", North Holland Publishing Company, Amsterdam (1976).

[Ci2] P.G.CIARLET, "Introduction à l'analyse numérique matricielle et à l'optimisation", Masson, Paris (1990).

[Du] G. DUVAUT, "Problèmes à frontière libre en théorie des milieux continus", Rapport de Recherche \# 185, LABORIA-IRIA, Rocquencourt (1976).

[GoTa1] R.L.V. GONZÁLEZ y D.A. TARZIA, "Optimization of heat flux in domain with temperature constraints", J.Optim.Th.Appl., 65 (1990), 245-256.

[GoTa2] R.L.V. GONZÁLEZ y D.A. TARZIA, "On some thermic flux optimization problems in a domain with Fourier boundary conditions and state restrictions", Mathematicae Notae, 34 (1990), 21-32.

[TaTa] E.D. TABACMAN y D.A. TARZIA, "Sufficient and/or necessary condition for the heat transfer coefficient on $\Gamma_{1}$ and the heat flux on $\Gamma_{2}$ to obtain a steady-state two-phase Stefan Problem", J. Diff. Eq., 77 (1989), 16-37.

[Ta] D.A. TARZIA, "Etude de l'inéquation variationnelle proposée par Duvaut pour le problème de Stefan à deux phases, II", Bollettino dell’Unione Matematica Italiana, 2B (1983), 589-603. 